\title{
Variations and expression features of CYP2D6 contribute to schizophrenia risk
}

\author{
Liang Ma $\mathbb{D}^{1} \cdot$ Anna Shcherbina ${ }^{2} \cdot$ Sundari Chetty $\mathbb{D}^{1,3}$
}

Received: 17 June 2019 / Revised: 27 January 2020 / Accepted: 30 January 2020 / Published online: 11 February 2020

(c) The Author(s) 2020. This article is published with open access

\begin{abstract}
Genome-wide association studies (GWAS) have successfully identified 145 loci implicated in schizophrenia (SCZ). However, the underlying mechanisms remain largely unknown. Here, we analyze 1497 RNA-seq data in combination with their genotype data and identify SNPs that are associated with expression throughout the genome by dissecting expression features to genes (eGene) and exon-exon junctions (eJunction). Then, we colocalize eGene and eJunction with SCZ GWAS using SMR and fine mapping. Multiple ChIP-seq data and DNA methylation data generated from brain were used for identifying the causal variants. Finally, we used a hypothesis-free (no SCZ risk loci considered) enrichment analysis to determine implicated pathways. We identified 171 genes and eight splicing junctions located within four genes (SNX19, $A R L 6 I P 4, A P O P T 1$, and CYP2D6) that potentially contribute to SCZ susceptibility. Among the genes, CYP2D6 is significantly associated with SCZ SNPs in eGene and eJunction. In-depth examination of the CYP2D6 region revealed that a nonsynonymous single nucleotide variant rs 16947 is strongly associated with a higher abundance of CYP2D6 exon 3 skipping junctions. While we found rs133377 and other functional SNPs in high linkage disequilibrium with rs 16947 $\left(r^{2}=0.9539\right)$, histone acetylation analysis showed they are located within active transcription start sites. Furthermore, our data-driven enrichment analysis showed that CYP2D6 is significantly involved in drug metabolism of codeine, tamoxifen, and citalopram. Our study facilitates an understanding of the genetic architecture of SCZ and provides new drug targets.
\end{abstract}

\section{Introduction}

Schizophrenia (SCZ) is a debilitating, highly heritable, and polygenic psychiatric condition affecting roughly $1 \%$ of the population. While prior genome-wide association studies

Supplementary information The online version of this article (https:// doi.org/10.1038/s41380-020-0675-y) contains supplementary material, which is available to authorized users.

Liang Ma

liangma1@stanford.edu

Sundari Chetty

chettys@ stanford.edu

1 Department of Psychiatry and Behavioral Sciences, Stanford University School of Medicine, Stanford, CA 94305, USA

2 Department of Biomedical Informatics, Stanford University, Stanford, CA 94305, USA

3 Institute for Stem Cell Biology and Regenerative Medicine, Stanford University School of Medicine, Stanford, CA 94305, USA
(GWASs) have yielded inconsistent findings [1] and candidate gene studies have identified risk SNPs and haplotypes [2], with increased sample size and improved study design, recent GWAS have successfully identified 145 risk loci associated with SCZ [3]. While these findings have identified regions in the genome harboring SCZ genes, almost all of the regions include multiple genes that are located within the same recombination hotspot intervals, making it challenging to identify causal genes.

One essential approach to determining the function of the identified SNPs is to combine GWASs with gene expression in postmortem human brains. Recently, several studies have applied these strategies to the 108 loci identified by the PGC in 2014 [4]. Using genotyped RNA-seq data generated by the CommonMind Consortium from postmortem dorsolateral prefrontal cortex (DLPFC), splicing QTLs were found to be significantly enriched in SCZ risk loci [5]. The enrichment of SCZ loci was also observed in another independent study of postmortem brain DLPFC samples [6], suggesting that combining these analytical techniques provides important insights into the mechanisms underlying SCZ. 
Studies integrating eQTL and GWAS data have almost exclusively used quantified expression across multiple transcript features, including annotated genes as well as annotation-guided transcripts. Notably, junction calls from short RNA-seq reads are considerably more reliable than assembled transcripts [7]. However, only a limited number of studies employed exon-exon junctions to identify splicing transcripts that contribute to the underlying phenotypes $[6,8]$. Recently, skipping of exon 2 and exon 3 of AS3MT was identified to contribute to SCZ risk using 495 postmortem brain cohorts [9]. Using the same specimens, we also successfully identified that the risk allele of SCZ SNPs is strongly associated with splicing junctions between exon 8 and exon 10 of SNX19 [10].

Here, we leveraged genotype and brain expression data provided by the Genotype-Tissue Expression (GTEx) project to elucidate the functional properties and potential roles of eQTL and splicing expression features in the etiology of SCZ. Integrating the GTEx brain eQTLs with the most recent SCZ GWAS data [3], we evaluated specific effects of the identified SCZ risk variants on gene expression features in combination with epigenetics data, and examined how genes of expression features affect genetic networks. Splicing transcripts have long been predicted to play roles in disease development, but the role of splicing in SCZ remains poorly understood. We address this gap by systematically evaluating exon-exon junctions in the human brain. By colocalizing the results from our gene- and junction-level analyses, we identify the functional genomic variants and potential effective splicing transcripts underlying SCZ susceptibility.

\section{Materials and methods}

An overview of our workflow can be found in Fig. S1.

\section{RNA-seq of postmortem brain}

A total of 1497 human brain samples across 13 brain regions were used in this study (Tables 1 and S1). All of the postmortem brain samples were collected by the GTEx consortium. The sample procurement has been described previously [11]. Raw gene and exon-exon junction reads counts were retrieved from GTEx portal. Gene lengths were calculated using GENCODE v19 annotations [12]. We converted gene counts to RPKM values using the total number of aligned reads across the 22 autosomal chromosomes. Considering a median depth of 84 million reads in the sequencing (Table 1), we converted junction counts to RP80M values using the total number of aligned reads across the autosomal chromosomes, which can be interpreted as the number of reads supporting the junction in an average library size [6].

\section{Genotyping data}

Whole-genome sequencing (WGS) datasets were retrieved from dbGap upon authentication by the GTEx Consortium

Table 1 Associations of the splicing junctions with SCZ risk SNPs across the 13 brain regions.

\begin{tabular}{|c|c|c|c|c|c|c|c|c|c|}
\hline \multirow[t]{3}{*}{ Tissue } & \multirow[t]{3}{*}{$N$} & \multirow[t]{3}{*}{ Average mapped reads counts } & \multicolumn{3}{|l|}{ ARL6IP4 } & \multicolumn{2}{|l|}{ APOPT1 } & \multicolumn{2}{|l|}{ CYP2D6 } \\
\hline & & & \multicolumn{3}{|l|}{ rs1790121 } & \multicolumn{2}{|c|}{ rs 10431750} & \multicolumn{2}{|l|}{ rs133377 } \\
\hline & & & Exon_2.3.1 & Exon_2.3.2 & Exon_1.4 & Exon_2.4 & Exon_3.4 & Exon_3.4 & Exon_2.4 \\
\hline Amygdala & 88 & $83,912,256$ & $1.45 \mathrm{E}-05$ & $1.48 \mathrm{E}-10$ & 0.0002 & 0.0165 & 0.021 & 0.0003 & $3.79 \mathrm{E}-06$ \\
\hline $\mathrm{ACC}$ & 109 & $88,136,513$ & $3.16 \mathrm{E}-09$ & $1.00 \mathrm{E}-13$ & $2.39 \mathrm{E}-07$ & $6.72 \mathrm{E}-05$ & $2.40 \mathrm{E}-04$ & $4.99 \mathrm{E}-08$ & $5.34 \mathrm{E}-06$ \\
\hline Caudate & 144 & $86,167,404$ & $4.26 \mathrm{E}-12$ & $2.15 \mathrm{E}-26$ & $1.01 \mathrm{E}-07$ & 0.0046 & $9.52 \mathrm{E}-04$ & $4.69 \mathrm{E}-11$ & $8.47 \mathrm{E}-12$ \\
\hline Cerebellar & 125 & $89,274,842$ & $1.38 \mathrm{E}-10$ & $1.93 \mathrm{E}-25$ & 0.0024 & $1.66 \mathrm{E}-04$ & 0.0048 & $7.62 \mathrm{E}-13$ & $9.09 \mathrm{E}-19$ \\
\hline Cerebellum & 154 & $82,622,299$ & $5.23 \mathrm{E}-15$ & $6.19 \mathrm{E}-30$ & 0.0383 & 0.0092 & $7.67 \mathrm{E}-05$ & $4.02 \mathrm{E}-10$ & $1.47 \mathrm{E}-21$ \\
\hline Cortex & 136 & $82,272,249$ & $1.05 \mathrm{E}-09$ & $1.76 \mathrm{E}-26$ & 0.0003 & $3.78 \mathrm{E}-05$ & $2.76 \mathrm{E}-04$ & $3.01 \mathrm{E}-07$ & $5.20 \mathrm{E}-05$ \\
\hline DLPFC & 118 & $85,916,703$ & $9.66 \mathrm{E}-15$ & $2.85 \mathrm{E}-22$ & $2.70 \mathrm{E}-05$ & $3.86 \mathrm{E}-04$ & $1.00 \mathrm{E}-04$ & $2.65 \mathrm{E}-04$ & $1.93 \mathrm{E}-06$ \\
\hline Hippocampus & 111 & $82,487,941$ & $5.95 \mathrm{E}-09$ & $2.64 \mathrm{E}-23$ & $1.26 \mathrm{E}-06$ & 0.0554 & 0.3127 & $3.02 \mathrm{E}-06$ & 0.011 \\
\hline Hypothalamus & 108 & $85,014,817$ & $2.84 \mathrm{E}-11$ & $3.45 \mathrm{E}-15$ & $8.28 \mathrm{E}-04$ & 0.011 & $5.07 \mathrm{E}-03$ & $1.42 \mathrm{E}-08$ & $6.22 \mathrm{E}-08$ \\
\hline Nucleus & 130 & $88,584,649$ & $7.08 \mathrm{E}-10$ & $6.76 \mathrm{E}-16$ & $8.77 \mathrm{E}-11$ & 0.0022 & $6.13 \mathrm{E}-05$ & $2.38 \mathrm{E}-12$ & $1.25 \mathrm{E}-13$ \\
\hline Putamen & 111 & $85,791,599$ & $5.78 \mathrm{E}-05$ & $4.97 \mathrm{E}-21$ & $8.40 \mathrm{E}-04$ & 0.8895 & 0.9736 & $3.72 \mathrm{E}-06$ & $3.29 \mathrm{E}-06$ \\
\hline Spinal & 83 & $82,583,948$ & $4.79 \mathrm{E}-08$ & $4.11 \mathrm{E}-14$ & $1.92 \mathrm{E}-04$ & 0.0889 & 0.0568 & 0.0917 & $1.93 \mathrm{E}-04$ \\
\hline Sub & 80 & $80,973,047$ & 0.0003 & $1.37 \mathrm{E}-14$ & 0.0022 & 1 & 0.6795 & $3.35 \mathrm{E}-05$ & $1.06 \mathrm{E}-04$ \\
\hline
\end{tabular}

False discovery rate (FDR) values are listed. Exon_8.10 in SNX19 was characterized in our prior work [10].

ACC anterior cingulate cortex (BA24), Caudate caudate (basal ganglia), Cerebellar cerebellar hemisphere, DLPFC dorsolateral prefrontal cortex (BA9), Nucleus nucleus accumbens (basal ganglia), Putamen putamen (basal ganglia), Spinal spinal cord (cervical c-1), Sub substantia nigra. 
(Accession: phs000424.v7.p2). We extracted a total of 42,585,769 genomic variants which were then filtered stepby-step by using PLINK 1.9 [13] if they: (1) had a genotype missing rate $>10 \%$ (272,734 variants); (2) had minor allele frequencies $<1 \%(31,110,395$ variants); and (3) deviated from Hardy-Weinberg equilibrium ( $p$ value $<1 \mathrm{E}-5$, 791,170 variants). Finally, we retained 10,411,470 variants for further analysis.

\section{cis-acting $\mathrm{eQTL}$ analysis}

cis-eQTL association was implemented separately by feature type (gene and junction) using Matrix eQTL R package [14] with the additive linear model, treating $\log 2$ transformed expression levels of each measurement (RPKM and RP80M) as the outcome. Features without expression (average counts $<0$ ) were excluded before eQTL analysis. To control for potential confounding factors, we adjust for ancestry (first three principle components (PCs) from the genotype data) [15], sex, and the first K PCs of the normalized expression features, where $\mathrm{K}$ was calculated separately by feature type using the sva Bioconductor package (gene: 13 PCs, junction: 13 PCs). False discovery rate (FDR) was assessed using the Benjamini-Hochberg algorithm (BH) across all cis-eQTL tests within each chromosome. We considered all variant-gene pairs (expression features to genes, eGene) and variant-junction pairs (eJunction) when the distance between features and SNP is $<1 \mathrm{MB}$.

\section{Colocalization of GWAS and eQTL associations}

In order to assess the probability that molecular traits as estimated by cis-eQTLs and physiological traits as estimated by GWAS share the same causal variant, we colocalized 8,171,061 SCZ GWAS summary statistics [3] with our eGene and eJunction results. We used SMR and HEIDI tests for the colocalization analysis [16]. We used the default parameters and performed for the genes and junctions. SNPs with linkage disequilibrium (LD) $r$-squared between top-SNP $>0.90$ or $<0.05$ were excluded as well as one of each pair of the remaining SNPs with LD r-squared $>0.90$. In addition, we conducted fine mappings of eGene and eJunction with SCZ GWAS separately. In the mapping process, any variants without either eQTL or GWAS association statistics were excluded.

\section{SNP annotation}

ANNOVAR [17] was used for characterizing the categories of variants which include exonic, upstream, downstream, $3^{\prime}$ UTR, 5'-UTR, intronic, and intergenic regions. Roadmap/ ENCODE2 chromatin-state signatures using a multivariate
Hidden Markov Model from brain tissue and cell types were extracted and visualized using WashU Epigenome Browser. For the identification of the binding locations of transcription factors (TF), ENCODE TF binding data was downloaded [18]. Then, we used bedtools intersect [19] to match SNPs to ChIP-seq peaks. Brain histone ChIP-seq data were obtained from the ENCODE portal. In addition, histone acetylation QTLs data (H3K9Ac ChIP-seq) generated from DLPFC of 433 individuals and DNA methylation QTLs data generated from 468 individuals were obtained from Brain xQTL Server [20].

\section{Functional enrichment}

We used three tools (WebGestalt [21], DAVID [22], and gProfiler [23]) for overrepresentation enrichment analysis which help us identify biological pathways that are significantly enriched in a gene list. The transcript features were mapped to Entrez Gene IDs and subsequently to KEGG pathway. Gene ontologies (GO) biological process and GO molecular function [24] were also calculated. FDR $(\mathrm{BH})$ and fold enrichment were imputed. FDR $<0.001$ was used as threshold.

\section{Results}

\section{Transcriptome-wide association study}

Alterations in the brain have been demonstrated to underlie cognitive deficits associated with SCZ, including impairments in working memory and cognitive flexibility [25]. To better understand the genetic interactions between expression features and genomic variations, we analyzed RNA-seq data of 13 brain regions from 1497 postmortem brains in combination with WGS data. We comprehensively identify cis-QTLs and expression features (gene and exon-exon splicing junctions) in the human brain with qualitycontrolled SNP genotyping data from the same individuals using Matrix eQTL [14] in a genome-wide manner (see Methods for details). To conservatively define eGene and eJunction SNPs, we applied BH procedure for multiple testing implemented in Matrix eQTL to the $p$ values. To evaluate the extent of overlap between eQTL and GWAS signatures in SCZ and to identify putative causative genes from GWAS associations, we then performed colocalization analyses by SMR methods using default parameters. We also performed fine mapping for an in-depth investigation. After performing these procedures, we identified a total of 55 genes (SMR) and 89 genes (mapping) in eGene (Tables S2 and S3) and 186 junctions within 78 genes (SMR) and 343 junctions within 133 genes in eJunction (mapping) (Tables S4 and S5) across the 13 brain regions (Figs. 1, S2, 

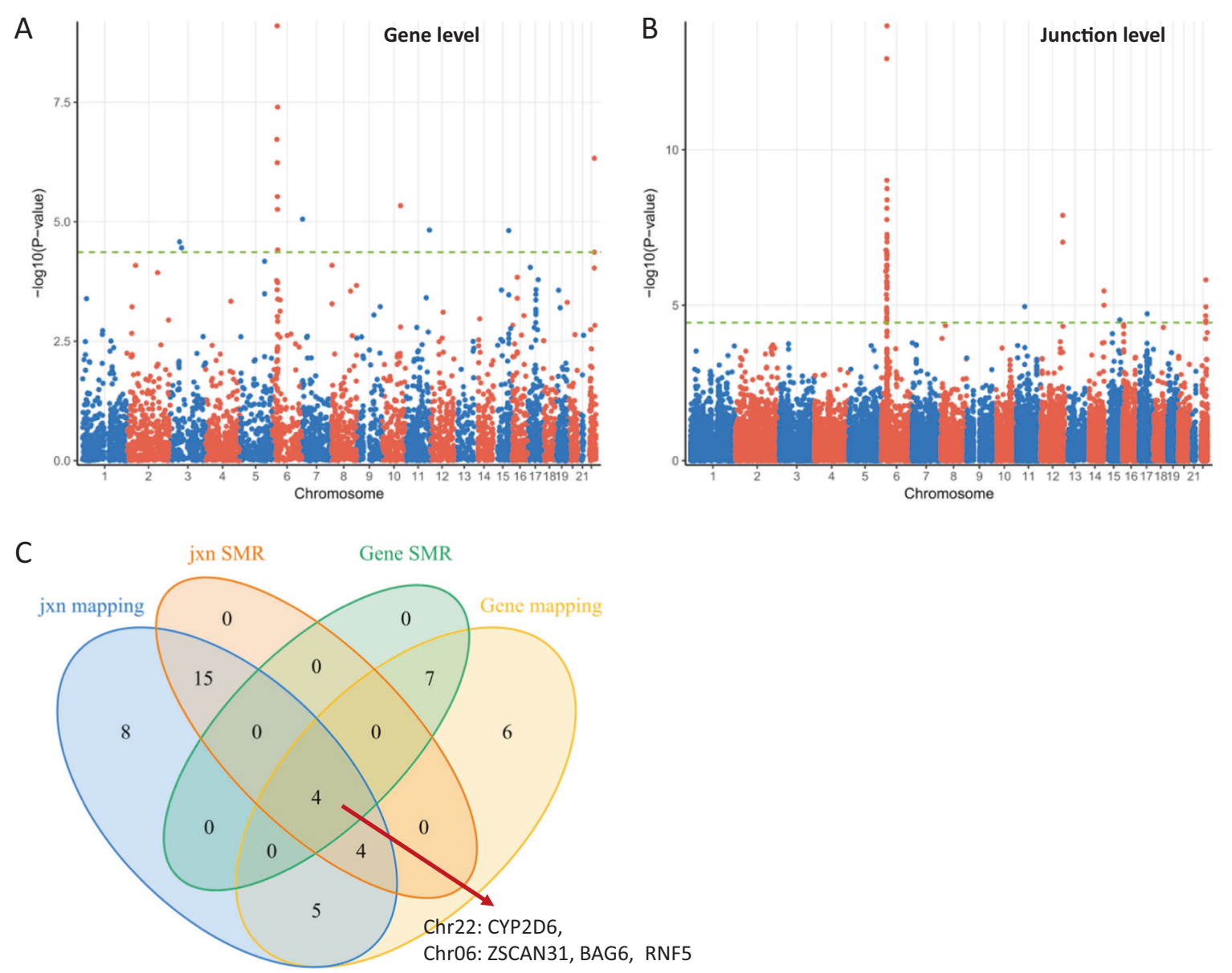

Fig. 1 Identification of CYP2D6 as a top candidate for schizophrenia risk. Manhattan plot of DLPFC (Brodmann Area 9) in gene level (a) and junction level (b). c Venn diagram of significant eGene and eJunction in dorsolateral prefrontal cortex (BA9). CYP2D6 was

and S3). Similar patterns of abundance of Cytochrome P450 2D6 (CYP2D6) gene Exon_2.4 and Exon_3.4 were observed across brain regions (Fig. S4) and developmental stages (Fig. S5), which is consistent with the functional connectivity between these brain regions [26].

As expected, most of the signals are from the major histocompatibility complex (MHC) region: 32/55 (SMR) and 51/89 (mapping) genes within eGene (Table S2 and Fig. 1a), and 41/78 and 74/133 (mapping) genes within eJunction (Tables S2-S5). The MHC is located in chromosome $6 \mathrm{p} 21$ and contains crucial regulators of the immune response. The MHC is the most gene dense and most polymorphic region of the human genome [27]. Complement component 4 (C4) structural variation was recently demonstrated to be related to the expression of $\mathrm{C} 4 \mathrm{~A}$ and $\mathrm{C} 4 \mathrm{~B}$ in postmortem brain [28]. In the current study, while $\mathrm{C} 4 \mathrm{~A}$ was detected in three brain regions in gene level by both SMR and fine mapping, C4A was also determined across ten brain regions in junction level by the two methods (Figs. 1b, c and S3 and Tables S4 and S5). observed in all overlapped combinations. Jxn, exon-exon junction. Manhattan plots and Venn diagrams of other 12 brain regions are shown in Supplementary Figs. S2, S3, and S8.

\section{Determination of splicing junctions}

Alternative pre-mRNA splicing is a regulated process that results in a single gene coding for multiple proteins by including or excluding particular exons of a gene. This generates spliced mRNAs that direct the synthesis of a diverse set of proteins with varied biological functions. To illuminate our understanding of SCZ risk in splicing junction level, we systematically evaluated the exon-exon junctions in the human brain. We found eight splicing junctions located within four genes: Exon_2.3.1 and Exon_2.3.2 in ARL6IP4; Exon_1.4, Exon_2.4, and Exon_3.4 in APOPT1; Exon_3.4 and Exon_2.4 in CYP2D6 (Fig. 2 and Table 1), including Exon 8.10 in $S N X 19$ as previously reported [10]. These junctions tag potential transcripts with alternative exonic boundaries (ARL6IP4 Exon_2.3.1 and Exon_2.3.2) or exon skipping (CYP2D6 Exon_3.4, SNX19 Exon_8.10, and APOPT1 Exon_1.4 and Exon_2.4) (Fig. 2). In prior work, we have systematically characterized splice junctions between exon 8 and exon 10 in SNX19 [10]. 
Fig. 2 Splicing junctions on gene structures. Red bar at chromosomes indicates the gene physical position on the chromosome. Green boxes represent exons; blue boxes represent untranslated regions; solid yellow lines indicate introns; yellow arrows on yellow lines indicate gene transcriptional directions. Red arrows indicate identified junctions: Exon_2.3.1 (chr12:123465814-123466117) and Exon_2.3.2 (chr12:123465847-123466117) in ARL6IP4;

Exon_1.4

(chr14:104029462-104053610),

Exon_2.4 (chr14:

104038158-104053610), and

Exon_3.4

(chr14:104040508-104053610) in

APOPT1; and Exon_3.4

(chr22:42524947-42525034) and

Exon_2.4 (chr22:

42522995-42523448) in

CYP2D6. The association of

SNX19 splicing junction

Exon_8.10 has been previously characterized in our prior work

[10]. Relative position of

CYP2D6 SNPs and histone

marker are indicated.
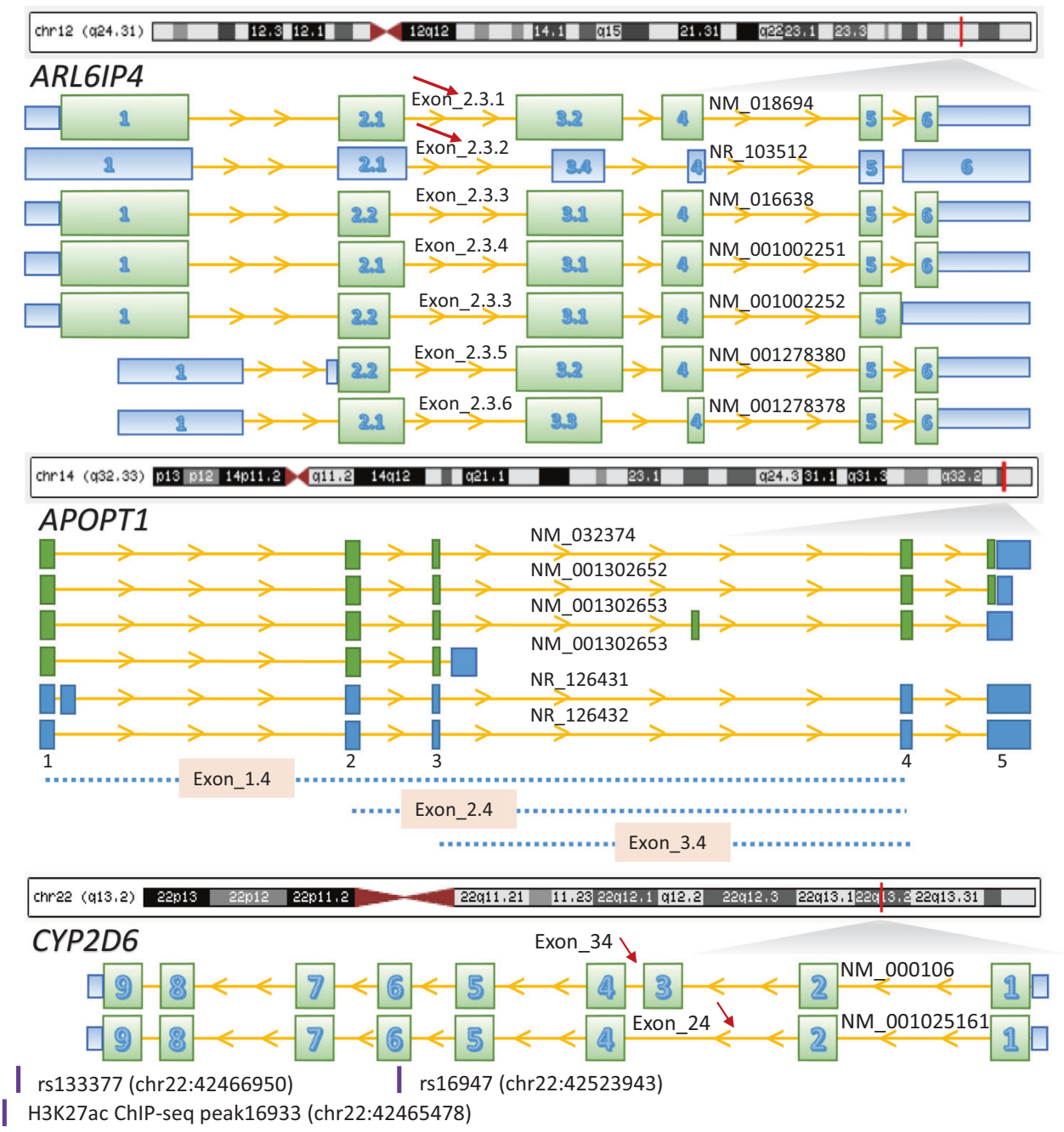

\section{Effects of schizophrenia genetic risk on junctions}

To determine how SCZ GWAS relates to feature expression, we next analyzed the trends of the associations. While SCZ risk allele is associated with down regulation of APOPT1 junction Exon_3.4, all other risk alleles are associated with up regulation of junctions (Fig. S6). Interestingly, SCZ risk allele is associate with up regulation of APOPT1 junction Exon_2.4 (Fig. 3). Opposing effects of SCZ risk allele on APOPT1 Exon_3.4 and the other two junctions indicate their diverse functions. These robust effects are reproduced in the DLPFC and many other brain regions (Table 1). Based on the genomic recombination rate, the GWAS-eQTL loci are in a linkage block where recombination is not estimated to occur. In addition, a cluster of SNPs is in high LD with top SCZ GWAS-eQTL SNPs: ARL6IP4 rs1790121, APOPT1 rs10431750, and CYP2D6 rs133377 (Fig. S7).

Strikingly, we found CYP2D6 to be the core gene in 8 out of the 13 brain regions determined in both eGene and eJunction across the 13 brain regions using both SMR and fine mapping (Figs. 1, S2, S3, S8 and Tables S2-S5). Differential expression levels of CYP2D6 between SCZ and controls in neurons and oligodendrocytes isolated from postmortem brains were comparable across the two groups (Supplementary Fig. S9), suggesting that alternative splicing of the gene rather than total gene expression levels is associated with $\mathrm{SCZ}$ risk. Therefore, we further investigated the CYP2D6 region. While multiple junctions were determined to be significant at the junction level across the 13 brain regions, we also found Exon_2.4, Exon_3.4, and Exon_7.8 to be significantly associated with SCZ risk SNPs (Figs. 3a-c, S10-S12 and Table S6). Interestingly, splicing occurred between exon 2 and exon 4 which resulted in maintaining or skipping exon 3. Exon_7.8 was included in all CYP2D6 transcripts which represents gene level (Fig. 2).

\section{Functional characterization of eJunction SNPs in SCZ}

Determining the underlying causal variants of complex disorders can be challenging because of the complex LD patterns between SNPs. We next attempted to functionally 
A

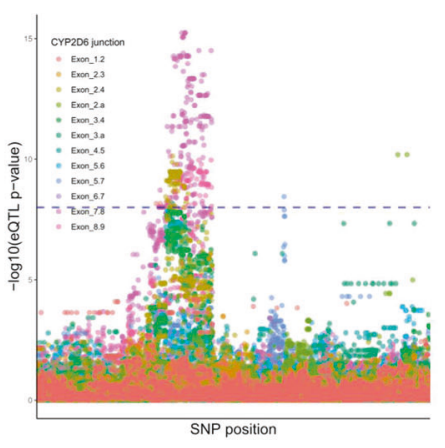

B

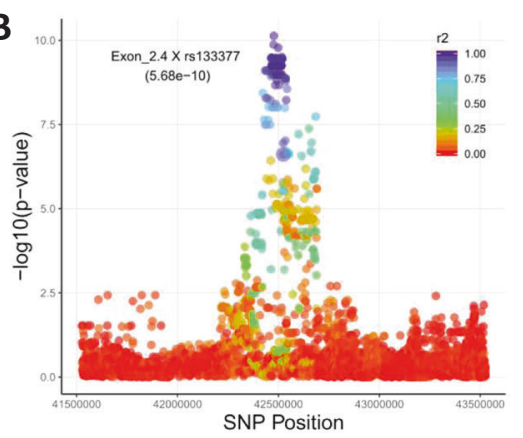

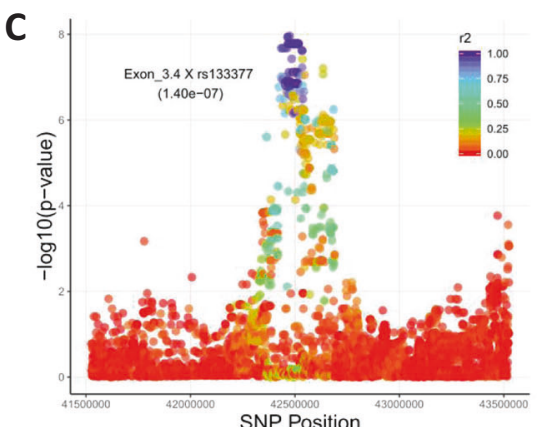

D

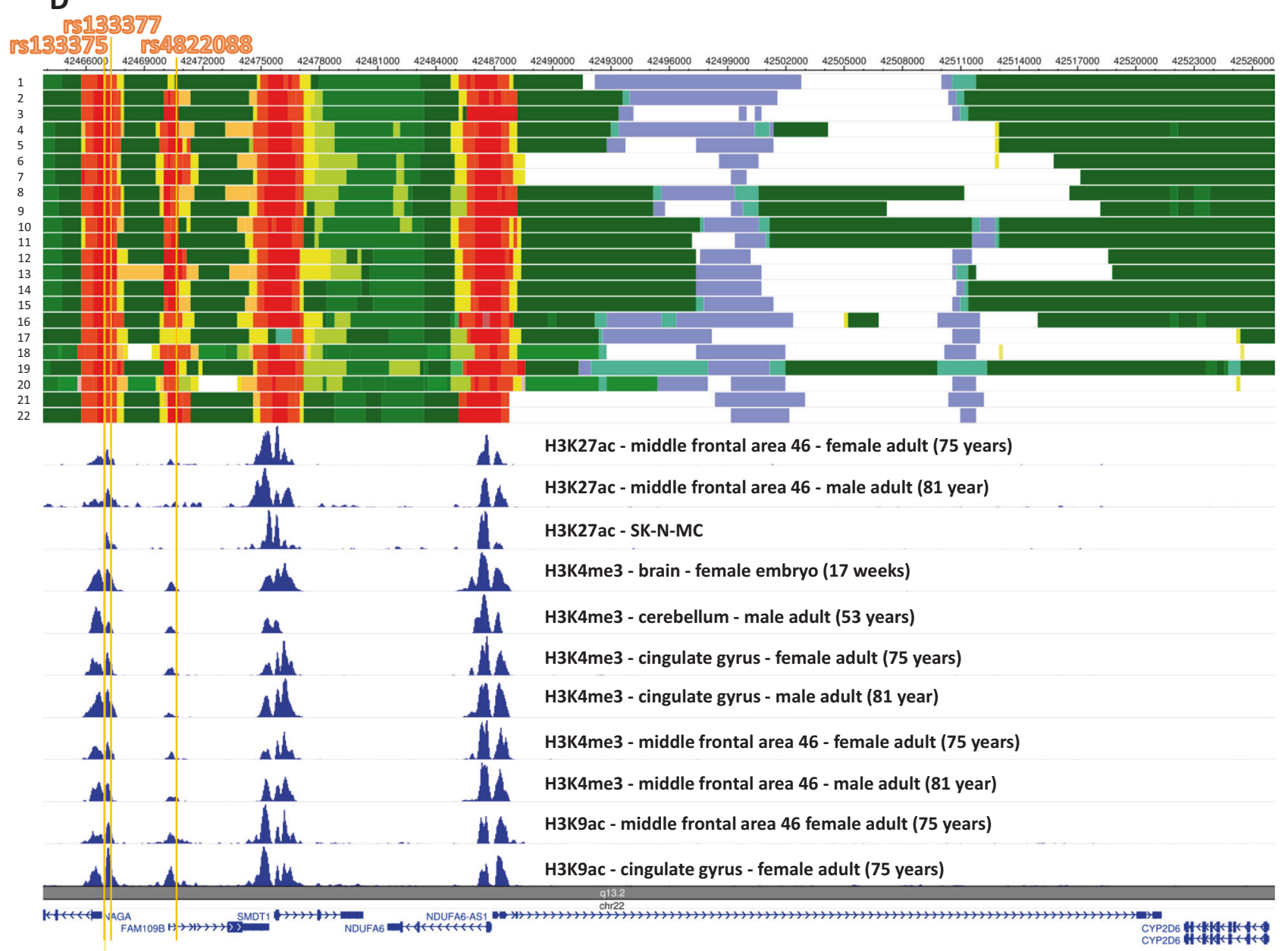

Fig. 3 Identification of functional variants of CYP2D6. a Association of SNP-junction pairs (eJunction) of CYP2D6. Association of junctions Exon_2.4 (b) and Exon_3.4 (c) with SNPs upstream and downstream of rs133377. $r^{2}$ was estimated using DLPFC samples. See association results from other 12 brain regions in Supplementary Figs. S10-S12. d Schizophrenia splicing SNPs are located within active transcription start sites. Upper section: ENCODE chromatin activation states in human brain from cortex-derived primary cultured neurospheres (1), angular gyrus $(2,3)$, anterior caudate $(4,5)$, cingulate gyrus $(6,7)$, germinal matrix $(8)$, hippocampus middle $(9,10)$, inferior temporal lobe $(11,12)$, dorsolateral prefrontal cortex $(13,14)$, substantia nigra $(15,16)$, fetal brain male $(17,18)$, fetal brain female $(19,20)$, NH-A astrocytes primary cells $(21,22)$. See details in Supplementary Table S7. Middle section: ChIP-seq of H3K27ac, H3K4me3, and H3K9ac. Lower section: reference genes. Yellow bars represent coordinates of rs133375, rs133377, and rs4822088. characterize eQTL SNPs by classifying the 255 SNPs in SCZ GWAS eJunction according to the definition in ANNOVAR [17]. As expected, most of the SNPs are nonexonic which accounts for $85.25 \%$ in SCZ GWAS
eGene and $87.09 \%$ in SCZ GWAS eJunction (Fig. S13 and Table S6). There are a total of seven exonic single nucleotide variant (SNV), four of which are nonsynonymous. Only one SNV was found in our target genes, which 
is, rs16947, located within exon 6 of CYP2D6 gene (NM_000106) (Fig. 2 and Table S6). Our CYP2D6 regional (2MB, about 5000 SNPs) LD analysis showed that SNPs in high LD with rs16947 are strongly associated with the abundance of splicing junctions (Exon_3.4 and Exon_2.4) in DLPFC and other brain regions (Table S6).

Enhancers have emerged as key cis-regulatory elements that play important regulatory roles in gene transcription, and they often reside distally from their target of regulation [29]. Using ENCODE chromatin activation states from brain, we found that SCZ splicing QTLs are located within active transcription start sites (Fig. 3d). Of the three SCZ splicing SNPs, rs133377 acts as an active transcription state across all the brains (Table S7). Three histone marks on H3K27ac, H3K4me3, and H3K9ac have been established to be associated with active transcription [30,31]. As expected, the functional SNPs we identified are located within the chromatin modification peaks (Fig. 3d). Note that the functional SNPs are in high LD with rs $16947\left(r^{2}=\right.$ 0.9539) (Fig. S14 and Table S6). In addition, we analyzed another independent H3K9ac ChIP-seq data and found rs133377 is significantly associated with a histone acetylation site, peak16933 in a $2 \mathrm{MB}$ sliding window (chr22:42465478, $p$ value $=3.78 \mathrm{E}-8$ ) (Fig. S15 and Table S8). While Exon_2.4 and Exon_4.5 in CYP2D6 and Exon_7.8 in CYP2D7P1 were determined to be significantly associated with rs 133377 in DLPFC, only Exon_2.4 is involved in splicing events. Using DNA methylation data of postmortem DLPFC brain from 468 individuals [20], we found $\mathrm{CpG}$ sites scattered around CYP2D6 are significantly interacted with both functional SNPs (e.g., rs133377) and chromatin (e.g., peak16933) (Table S8), implying they function together to regulate CYP2D6 gene transcription. Additionally, we characterized the SNPs that are interacted with chromatin status and CpG sites, and find that the SNPs (e.g., rs133377) are overlaid with zinc finger motifs (e.g., ZNF263) and other TF binding sites (Table S9 and Fig. S16). Zinc finger motifs have known methyltransferase functions [32], implying the variants may influence gene expression by disrupting methyltransferase enzymes' ability to recognize their motifs.

\section{Enrichment analysis of eJunction}

To further verify our systematic genomics results and gain mechanistic insight, we next took an unbiased data-driven approach and performed a comprehensive gene-set pathway enrichment analysis to explore the potential functional implications of the genome-wide significant genes overlapped in junction level and gene level using WebGestalt, DAVID, and gProfiler across the 13 brain regions. An average of 822 genes from the 13 brain regions was determined and used for the enrichment analysis, and more than 300 pathways were imputed for each brain region by the three tools. Two top pathways that include CYP2D6 are associated with metabolism of xenobiotics by cytochrome P450 (hsa00980) and drug metabolism (hsa00982) and were consistently identified by each of the three tools across the 13 brain regions (Figs. $4 \mathrm{a}-\mathrm{c}$ and S17-S19 and Tables S10-S12). When we took an in-depth examination of the KEGG hsa00982 pathway, we found that CYP2D6 was associated with the metabolic process of psychiatric drugs: tamoxifen, codeine, and citalopram (Figs. 4d and S17-S19). Similarly, GO biological process analyses showed CYP2D6 and related genes play a role in steroid metabolic process in DLPFC and other brain regions (GO:0008202) (Table S13). GO molecular function analyses showed CYP2D6 and 13 related genes involved in drug binding (GO:0008144) in DLPFC and other brain regions (Table S14).

\section{Discussion}

In this study, we analyzed a large-scale transcriptome derived from 1497 human postmortem brain samples in combination with WGS data. SCZ is a complex genetic disorder and over a hundred loci have been identified. However, it remains unclear how genetics affect the abundance of expression features. We identified potential effective splicing transcripts underlying SCZ susceptibility using the most recent GTEx brain RNA-seq data (released on June 30, 2017) [11] and 2018 SCZ GWAS summary statistics [3]. We identify CYP2D6 to be an important candidate gene through several unbiased strategies: (1) by comparing gene overlapping using SMR and fine mapping in gene and junction level across 13 brain regions; and (2) through hypothesis-free overrepresentation enrichment analysis of eJunction genes. Furthermore, by analyzing multiple sources of ChIP-seq data generated from postmortem brains, we identify causal SNPs associated with CYP2D6.

Our principal findings include four major observations. First, we have identified a total of 171 genes across 13 brain regions for which genetic variation for expression colocalize with genetic variation for $\mathrm{SCZ}$ risk. Some differences in results are expected using alternative colocalization methods and references. Using 206 postmortem brain DLPFC samples from normal individuals, Takata et al. evaluated SCZ risk loci that are involved in splicing events based on assembled transcripts [5]. In the current study, we replicated reported genes, for example, SNX19, ARL6IP4, APOPT1 [5], $C 4 A$, and $C 4 B$ [28], and discovered new genes such as CYP2D6.

Second, we identified a list of exon-exon junctions that tag genetic transcripts. In prior work, most analyses focus 

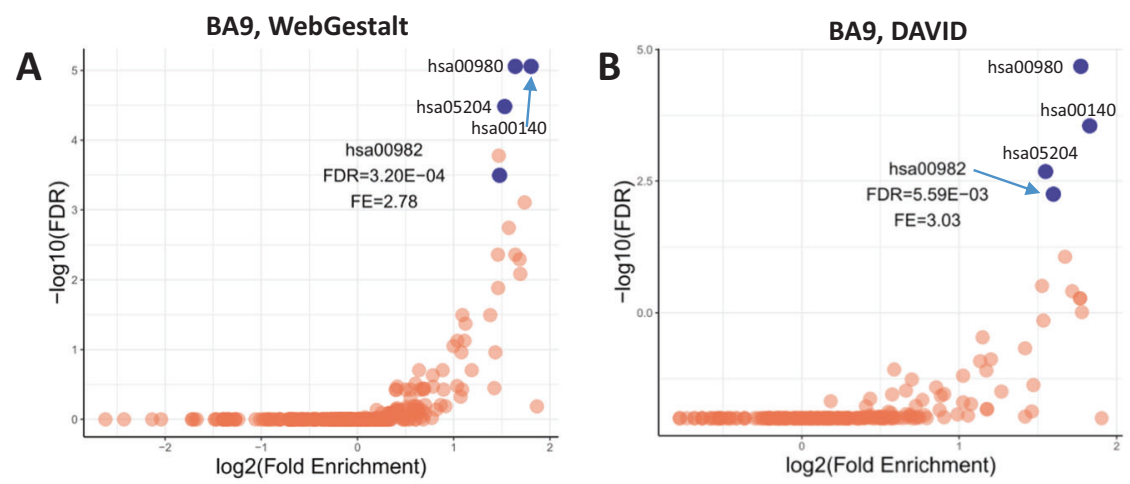

BA9, gProfiler

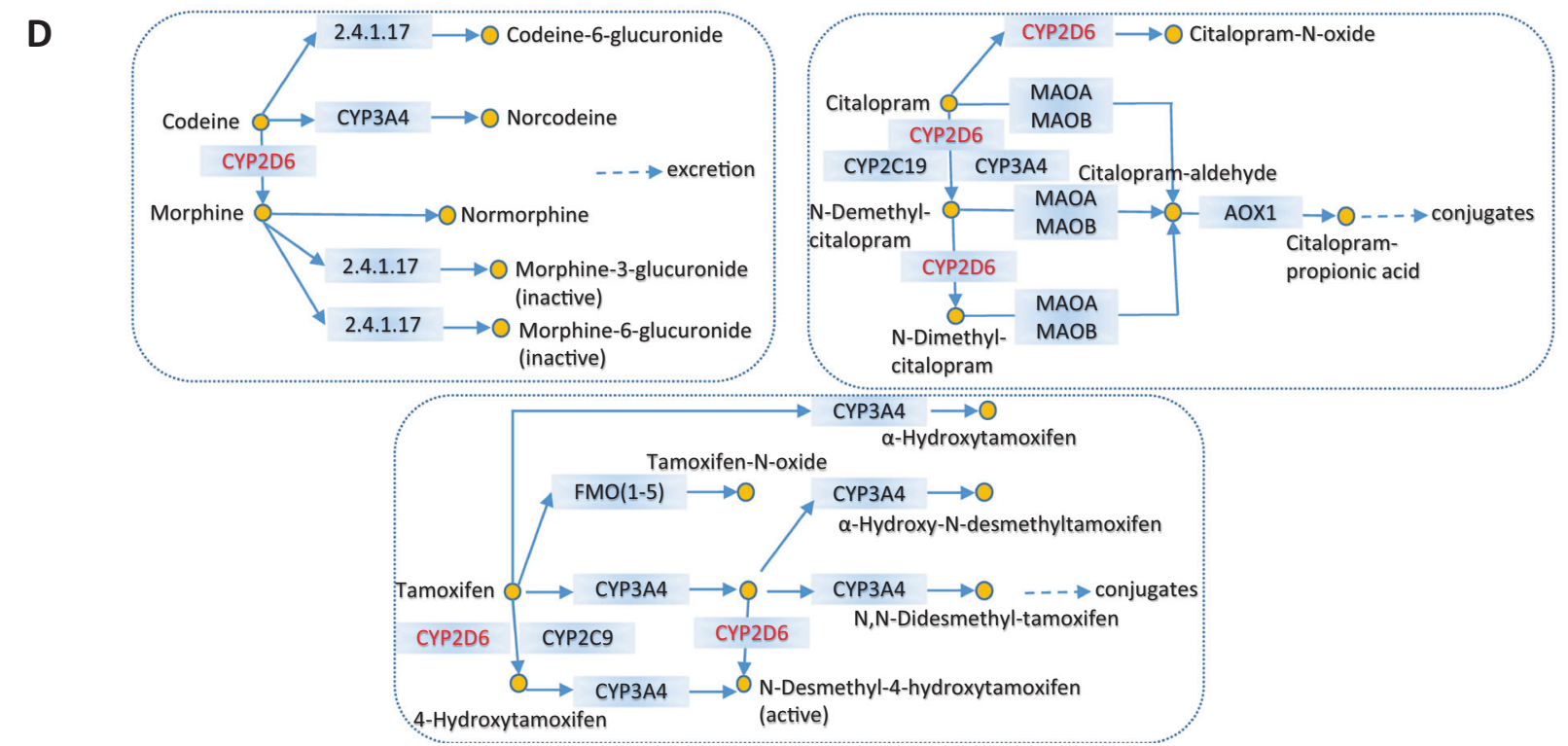

Fig. 4 Pathway analysis of overlapped significant genes of eJunctions and eGenes. KEGG pathways enriched for 798 genes that are both significant in gene level and junction level in dorsolateral prefrontal cortex (BA9) by using WebGestalt (a), DAVID (b), and gProfiler (c). Blue dots are overlapped top KEGG pathways in BA9 using WebGestalt, DAVID, and gProfiler. FDR false discovery rate.

almost exclusively on the marginal eQTL signal that typically represents the primary, or most significant, eQTL signal, rather than dissecting this signal into multiple independent features for each gene. While predicted transcripts are more likely to be used as a reference, we used exon-exon junctions to tag specific transcripts that considerably increase specificity. Our eJunction analyses identify eight splicing junctions that are located in four genes. Our recent study has systematically evaluated SNX19 Exon_8.10 transcripts and their potential susceptibility to SCZ [10]. APOPT1 was demonstrated to be located in mitochondria matrix, and involved in possessing an N-terminal mitochondrial targeting signal [33]. ARL6IP4 play a functional role in premRNA splicing [34, 35]. However, the mechanisms of APOPT1 and ARL6IP4 are still largely unknown.
Results of other 12 brain regions are shown in Supplementary Figs. S17 (WebGestalt), S18 (DAVID), and S19 (gProfiler). d CYP2D6 in the drug metabolism process of KEGG pathway hsa00982. 2.4.1.17: UGT1A(1,3-10), UGT2A(1-3), UGT2B $(4,7,10,11,17,28)$.

Third, we illustrated an underlying mechanism for SCZ risk. There is accumulating evidence that CYP2D6 is involved in the metabolism of clinically used drugs [36]. A clinical study reported that CYP2D6 plays an important role in controlling the state of aripiprazole in the plasma which has been established as a form of treatment for SCZ [37]. CYP2D6 has also been reported to have significant effects on psychotic symptoms [38] and cognitive performance [39] of SCZ patients following risperidone treatment. Our data-driven enrichment analysis identified CYP2D6 as a key component of the drug metabolism pathways. CYP2D6/codeine has been highlighted as an antidepressant gene/drug pair in clinical therapy by Clinical Pharmacogenetics Implementation Consortium [40]. Codeine is bioactivated into morphine by CYP2D6 to exert its analgesic effect. Morphine, a strong opioid agonist, acts directly on 
the central nervous system and has been strongly implicated in addiction and SCZ pathophysiology [41]. Citalopram is a selective serotonin reuptake inhibitor used to treat major depression disorders [42]. Tamoxifen is well-known as an estrogen receptor modulator commonly used to treat breast cancer. In addition, we observed CYP2D6 to be involved in the metabolism of neuroactive steroids which are present in human postmortem brain tissue. In fact, concentrations of neuroactive steroids are known to be altered in subjects with SCZ and bipolar disorder [43]. Overall, these findings highlight CYP2D6 as an important candidate for further biological investigation.

Finally, by characterizing the properties of the detected eJunction SNPs, we found a nonsynonymous SNV, rs16947, to be associated with abundance of splicing junctions related to its exon 3 skipping. This leads to an inframe deletion that shortens the translated protein by 51 amino acids. This risk allele of the SNV could result in an amino acid substitution that has been shown to reduce enzyme activity after recombinant cDNA transfection $[44,45]$. On the other hand, we found three SNPs, which are in strong LD with rs16947, to be located within active chromatin modification regions in the brain, indicating that they are actively involved in direct regulation of CYP2D6 gene transcription [30,31]. Thus, taken together, it is conceivable these alterations (changes in protein structure and/ or chromatin modifications) affect the transcriptional activity of $C Y P 2 D 6$, leading to downstream changes in gene expression, which could further alter enzyme activity in drug metabolism. This alternative pre-mRNA splicing may also contribute to the extensive variability in CYP2D6 activity observed across individuals [46].

Although the sample size in this study is substantial $(N$ $=1497$ ), the sample size of each brain region is relatively small $($ mean $=115)$, so increasing sample size could help identify additional brain functional SNPs and splicing junctions with increased confidence. In addition, CRISPR genome editing of the causal SNPs, such as rs133377, on hiPSC-derived neurons would be of great value, although the technologies are still largely challenging [47].

In summary, we comprehensively analyzed expression features that are mediated by genomic markers across the human brain regions, described the characteristics of these SNPs, and demonstrated that the list of brain SNPs can be used to identify plausible candidate transcripts/variants that are causally associated with SCZ. These findings will be of great use in generating new animal and cellular models for SCZ.

\section{Code availability}

PLINK 1.9, https://www.cog-genomics.org/plink/. Matrix eQTL R package, http://www.bios.unc.edu/research/
genomic_software/Matrix_eQTL/. sva Bioconductor package, https://bioconductor.org/packages/release/bioc/html/ sva.html. SMR, https://cnsgenomics.com/software/smr/ \#Overview. ANNOVAR, http://annovar.openbioinforma tics.org/en/latest/. WashU Epigenome Browser, https:// epgg-test.wustl.edu/browser/. GTEx portal, https:// gtexportal.org/home/datasets.

Acknowledgements We thank all the staff, especially John B. Hanks at the Stanford Research Computing Center, for their excellent computational support. We thank Yuxing Liao at Baylor College of Medicine and Hedi Peterson at University of Tartu for their suggestions and help. This work was supported by grants from the Stanford University School of Medicine and a Siebel Fellowship awarded to SC. Data were generated by the GTEx Project supported by the Common Fund of the National Institutes of Health.

Author contributions LM conceived the study, conducted the analysis, and wrote the draft. LM and SC collected the data. AS helped with data collection and analyses. SC provided intellectual input and financial support for the study. All authors contributed to the editing of the manuscript, and approved the final manuscript.

\section{Compliance with ethical standards}

Conflict of interest The authors declare that they have no conflict of interest.

Publisher's note Springer Nature remains neutral with regard to jurisdictional claims in published maps and institutional affiliations.

Open Access This article is licensed under a Creative Commons Attribution 4.0 International License, which permits use, sharing, adaptation, distribution and reproduction in any medium or format, as long as you give appropriate credit to the original author(s) and the source, provide a link to the Creative Commons license, and indicate if changes were made. The images or other third party material in this article are included in the article's Creative Commons license, unless indicated otherwise in a credit line to the material. If material is not included in the article's Creative Commons license and your intended use is not permitted by statutory regulation or exceeds the permitted use, you will need to obtain permission directly from the copyright holder. To view a copy of this license, visit http://creativecommons. org/licenses/by/4.0/.

\section{References}

1. Ma L, Tang J, Wang D, Zhang W, Liu W, Wang D, et al. Evaluating risk loci for schizophrenia distilled from genome-wide association studies in Han Chinese from Central China. Mol Psychiatry. 2013;18:638-9.

2. Ma L, Wu DD, Ma SL, Tan L, Chen X, Tang NL, et al. Molecular evolution in the CREB1 signal pathway and a rare haplotype in CREB1 with genetic predisposition to schizophrenia. J Psychiatr Res. 2014;57:84-9.

3. Pardinas AF, Holmans P, Pocklington AJ, Escott-Price V, Ripke $\mathrm{S}$, Carrera N, et al. Common schizophrenia alleles are enriched in mutation-intolerant genes and in regions under strong background selection. Nat Genet. 2018;50:381-9.

4. Schizophrenia Working Group of the Psychiatric Genomics Consortium. Biological insights from 108 schizophreniaassociated genetic loci. Nature. 2014;511:421-7. 
5. Takata A, Matsumoto N, Kato T. Genome-wide identification of splicing QTLs in the human brain and their enrichment among schizophrenia-associated loci. Nat Commun. 2017;8:14519.

6. Jaffe AE, Straub RE, Shin JH, Tao R, Gao Y, Collado-Torres L, et al. Developmental and genetic regulation of the human cortex transcriptome illuminate schizophrenia pathogenesis. Nat Neurosci. 2018;21:1117-25.

7. Steijger T, Abril JF, Engstrom PG, Kokocinski F, Consortium R, Hubbard TJ, et al. Assessment of transcript reconstruction methods for RNA-seq. Nat Methods. 2013;10:1177-84.

8. Ma L, Jia P, Zhao Z. Splicing QTL of human adipose-related traits. Sci Rep. 2018;8:318.

9. Li M, Jaffe AE, Straub RE, Tao R, Shin JH, Wang Y, et al. A human-specific AS3MT isoform and BORCS7 are molecular risk factors in the 10q24.32 schizophrenia-associated locus. Nat Med. 2016;22:649-56.

10. Ma L, Semick SA, Chen Q, Li C, Tao R, Price AJ, et al. Schizophrenia risk variants influence multiple classes of transcripts of sorting nexin 19 (SNX19). Mol Psychiatry. 2019. https://doi.org/ 10.1038/s41380-018-0293-0.

11. GTEx_Consortium, LaboratoryDA, Coordinating Center-Analysis Working G, Statistical Methods groups-Analysis Working G, Enhancing Gg, Fund NIHC, et al. Genetic effects on gene expression across human tissues. Nature. 2017;550:204-13.

12. Harrow J, Frankish A, Gonzalez JM, Tapanari E, Diekhans M, Kokocinski F, et al. GENCODE: the reference human genome annotation for the ENCODE Project. Genome Res. 2012;22:1760-74.

13. Chang CC, Chow CC, Tellier LC, Vattikuti S, Purcell SM, Lee JJ. Second-generation PLINK: rising to the challenge of larger and richer datasets. Gigascience. 2015;4:7.

14. Shabalin AA. Matrix eQTL: ultra fast eQTL analysis via large matrix operations. Bioinformatics. 2012;28:1353-8.

15. Price AL, Patterson NJ, Plenge RM, Weinblatt ME, Shadick NA, Reich D. Principal components analysis corrects for stratification in genome-wide association studies. Nat Genet. 2006;38:904-9.

16. Zhu Z, Zhang F, Hu H, Bakshi A, Robinson MR, Powell JE, et al. Integration of summary data from GWAS and eQTL studies predicts complex trait gene targets. Nat Genet. 2016;48:481-7.

17. Wang $\mathrm{K}$, Li M, Hakonarson H. ANNOVAR: functional annotation of genetic variants from high-throughput sequencing data. Nucleic Acids Res. 2010;38:e164.

18. Kheradpour P, Kellis M. Systematic discovery and characterization of regulatory motifs in ENCODE TF binding experiments. Nucleic Acids Res. 2014;42:2976-87.

19. Quinlan AR, Hall IM. BEDTools: a flexible suite of utilities for comparing genomic features. Bioinformatics. 2010;26:841-2.

20. Ng B, White CC, Klein HU, Sieberts SK, McCabe C, Patrick E, et al. An xQTL map integrates the genetic architecture of the human brain's transcriptome and epigenome. Nat Neurosci. 2017;20:1418-26.

21. Wang J, Vasaikar S, Shi Z, Greer M, Zhang B. WebGestalt 2017: a more comprehensive, powerful, flexible and interactive gene set enrichment analysis toolkit. Nucleic Acids Res. 2017;45:W130-7.

22. Huang da W, Sherman BT, Lempicki RA. Systematic and integrative analysis of large gene lists using DAVID bioinformatics resources. Nat Protoc. 2009;4:44-57.

23. Reimand J, Arak T, Vilo J. g:Profiler-a web server for functional interpretation of gene lists (2011 update). Nucleic Acids Res. 2011;39:W307-15.

24. Gene Ontology Consortium. Gene Ontology Consortium: going forward. Nucleic Acids Res. 2015;43:D1049-56.

25. Weinberger DR, Berman KF, Zec RF. Physiologic dysfunction of dorsolateral prefrontal cortex in schizophrenia. I. Regional cerebral blood flow evidence. Arch Gen Psychiatry. 1986;43:114-24.
26. Tanimizu T, Kenney JW, Okano E, Kadoma K, Frankland PW, Kida S. Functional connectivity of multiple brain regions required for the consolidation of social recognition memory. J Neurosci. 2017;37:4103-16.

27. Trowsdale J, Knight JC. Major histocompatibility complex genomics and human disease. Annu Rev Genomics Hum Genet. 2013;14:301-23.

28. Sekar A, Bialas AR, de Rivera H, Davis A, Hammond TR, Kamitaki N, et al. Schizophrenia risk from complex variation of complement component 4. Nature. 2016;530:177-83.

29. Heintzman ND, Ren B. Finding distal regulatory elements in the human genome. Curr Opin Genet Dev. 2009;19:541-9.

30. Creyghton MP, Cheng AW, Welstead GG, Kooistra T, Carey BW, Steine EJ, et al. Histone H3K27ac separates active from poised enhancers and predicts developmental state. Proc Natl Acad Sci USA. 2010;107:21931-6.

31. Gates LA, Shi J, Rohira AD, Feng Q, Zhu B, Bedford MT, et al. Acetylation on histone H3 lysine 9 mediates a switch from transcription initiation to elongation. J Biol Chem. 2017;292:14456-72.

32. Chaikind B, Ostermeier M. Directed evolution of improved zinc finger methyltransferases. PLoS ONE. 2014;9:e96931.

33. Melchionda L, Haack TB, Hardy S, Abbink TE, Fernandez-Vizarra E, Lamantea E, et al. Mutations in APOPT1, encoding a mitochondrial protein, cause cavitating leukoencephalopathy with cytochrome c oxidase deficiency. Am J Hum Genet. 2014;95:315-25.

34. Sasahara K, Yamaoka T, Moritani M, Tanaka M, Iwahana H, Yoshimoto K, et al. Molecular cloning and expression analysis of a putative nuclear protein, SR-25. Biochem Biophys Res Commun. 2000;269:444-50.

35. Li Q, Zhao H, Jiang L, Che Y, Dong C, Wang L, et al. An SRprotein induced by HSVI binding to cells functioning as a splicing inhibitor of viral pre-mRNA. J Mol Biol. 2002;316:887-94.

36. Phillips KA, Veenstra DL, Oren E, Lee JK, Sadee W. Potential role of pharmacogenomics in reducing adverse drug reactions: a systematic review. J Am Med Assoc. 2001;286:2270-9.

37. Suzuki T, Mihara K, Nakamura A, Kagawa S, Nagai G, Nemoto $\mathrm{K}$, et al. Effects of genetic polymorphisms of CYP2D6, CYP3A5, and $\mathrm{ABCB} 1$ on the steady-state plasma concentrations of aripiprazole and its active metabolite, dehydroaripiprazole, in Japanese patients with schizophrenia. Ther Drug Monit. 2014;36:651-5.

38. Bartecek R, Jurica J, Zrustova J, Kasparek T, Pindurova E, Zourkova A. Relevance of CYP2D6 variability in first-episode schizophrenia patients treated with risperidone. Neuro Endocrinol Lett. 2012;33:236-44.

39. Zeng L, Kang C, Yuan J, Zhang Y, Wei Y, Xu L, et al. CYP2D6 polymorphisms are associated with effects of risperidone on neurocognitive performance in schizophrenia. Schizophr Res. 2017;188:50-1.

40. Crews KR, Gaedigk A, Dunnenberger HM, Klein TE, Shen DD, Callaghan JT, et al. Clinical Pharmacogenetics Implementation Consortium (CPIC) guidelines for codeine therapy in the context of cytochrome P450 2D6 (CYP2D6) genotype. Clin Pharm Ther. 2012;91:321-6.

41. Stefano GB, Kralickova M, Ptacek R, Kuzelova H, Esch T, Kream RM. Low dose morphine adjuvant therapy for enhanced efficacy of antipsychotic drug action: potential involvement of endogenous morphine in the pathophysiology of schizophrenia. Med Sci Monit. 2012;18:HY23-26.

42. Cipriani A, Purgato M, Furukawa TA, Trespidi C, Imperadore G, Signoretti A, et al. Citalopram versus other anti-depressive agents for depression. Cochrane Database Syst Rev. 2012;11:CD006534.

43. Marx CE, Stevens RD, Shampine LJ, Uzunova V, Trost WT, Butterfield MI, et al. Neuroactive steroids are altered in schizophrenia and bipolar disorder: relevance to pathophysiology and therapeutics. Neuropsychopharmacol. 2006;31:1249-63. 
44. Marcucci KA, Pearce RE, Crespi C, Steimel DT, Leeder JS, Gaedigk A. Characterization of cytochrome P450 2D6.1 (CYP2D6.1), CYP2D6.2, and CYP2D6.17 activities toward model CYP2D6 substrates dextromethorphan, bufuralol, and debrisoquine. Drug Metab Dispos. 2002;30:595-601.

45. Yu A, Kneller BM, Rettie AE, Haining RL. Expression, purification, biochemical characterization, and comparative function of human cytochrome P450 2D6.1, 2D6.2, 2D6.10, and 2D6.17 allelic isoforms. J Pharm Exp Ther. 2002;303:1291-300.

46. Zanger UM, Raimundo S, Eichelbaum M. Cytochrome P450 2D6: overview and update on pharmacology, genetics, biochemistry. Naunyn Schmiedebergs Arch Pharm. 2004;369:23-37.

47. Wen Z. Modeling neurodevelopmental and psychiatric diseases with human iPSCs. J Neurosci Res. 2017;95:1097-109. 